\title{
Mujer y Ciencia Política en Chile: ¿algo nuevo bajo el sol?*
}

\author{
Ma. de los Ángeles FERNÁNDEZ ${ }^{1}$ \\ "La Ciencia Política, como disciplina, ha sido notoria \\ en su relativa carencia de receptividad a las mujeres como \\ cientistas políticas y al estudio de la mujer en política". \\ (Cook, citada en Kelly, Williams y Fisher, 1994).
}

\section{Introducción}

$\mathrm{L}$

as mujeres son minoría en las comunidades de Ciencia Política en los distintos contextos nacionales. El argumento más recurrente para entender esta situación es de corte estructural: se debería a la gestalt particular de la disciplina en sí misma, que conduce y apoya un cierto tipo de comprensión que limita tanto la naturaleza de la investigación como las conclusiones a las que es posible desembocar (Guittens, 1983). Otras explicaciones para el reducido número de mujeres apuntan a que éstas no serían bien recibidas en una materia tan dominada por el hombre (Chapman, 1996). Ferguson (1987) lo plantea claramente al indicar que el problema de ingreso de la mujer, como objeto de estudio y como sujeto profesional, en la Ciencia Política puede destacarse en dos dimensiones: la epistemología dominante y la política profesional. Lo concreto es que muchos ensayos críticos han demostrado que los cientistas políticos, de manera regular, excluyen de su trabajo cualquier discusión sobre la mujer y, lo que es más, la Ciencia Política aparecería incluso más masculina que otras disciplinas de las Ciencias Sociales y Humanidades (Sarkees

\footnotetext{
* Mucho me han servido los comentarios de Cecilia Osorio, Stéphanie Alenda, Jane Jaquette y Alfredo Joignant. Sin embargo, el resultado final es de mi exclusiva responsabilidad.

Agradezco a Santiago Edwards y a Antonio Peñafiel la recopilación de los datos contenidos en el presente artículo, para el ámbito de la docencia.

1. Cientista política, Doctora (c) en Procesos Políticos Contemporáneos, Universidad de Santiago de Compostela. Ex presidenta de la Asociación Chilena de Ciencia Política (20002002), actualmente es investigadora del Instituo de Ciencias Sociales, Universidad Diego Portales. Coinvestigadora del proyecto Ecos-Conicyt "Permanencia y cambios del compromiso político en las democracias representativas" y del proyecto Fondecyt No 1060479 "Evolución histórica y determinantes sociales, étnicas, culturales y coyunturales del comportamiento electoral de los chilenos 1989-2005". Correo electrónico: angeles.fernandez@udp.cl
} 
y McGlen, 1992; Kelly, Williams y Fisher, 1994; Kenney, 1995; Mathews y Andersen, 2001). Sin embargo, también es justo reconocer el avance experimentado en las últimas décadas, ya que la nueva voz feminista ha logrado instalarse en la disciplina, gracias especialmente a los aportes realizados desde el campo de la teoría política.

¿Cuál es el panorama que observamos en Chile? Nada muy diferente al de otros contextos. Si bien no existen estudios sistemáticos al respecto, una primera indagación de los programas de estudio y la docencia impartida a nivel de pregrado ratifica la dominación masculina en la Ciencia Política chilena, con sólo un porcentaje de 24,5\% de mujeres que ejercen docencia (Fuentes y Santana, 2005). Sin embargo, en la fase de entrada a la disciplina, se comienza a percibir un notorio interés en las mujeres por estudiar Ciencia Política.

La pregunta es pertinente por varios motivos: el primero, de carácter académico, dado el avance internacional del campo de estudios especializado denominado "Género y política" al interior de la disciplina, así como por experimentar ésta en Chile un paradójico punto de inflexión caracterizado por un incremento sostenido de la oferta de enseñanza de pregrado, la realización del Congreso Mundial de Ciencia Política en el año 2009, una significativa visibilidad en los medios de comunicación, insuficientes recursos para la investigación, todo ello acompañado por una demanda creciente de profesionalización (Fernández, 2004, 2005; Altman, 2005); el segundo, por sucesos políticos y posibles cambios culturales más amplios: por primera vez en Chile una mujer, Michelle Bachelet, es electa Presidenta de la República, la que ha conformado su gabinete en base al criterio de la paridad de género. Esta novedosa situación académica y política interpela a la Ciencia Política con múltiples preguntas que, hasta el momento, no ha estado en capacidad de responder de manera eficiente, habida cuenta de la ausencia de una reflexión sistemática acerca de la relación entre el género y los fenómenos de poder en el contexto nacional.

Por otro lado, la dimensión de género de una profesión y sus implicancias constituye una parte de la modernización y de los efectos democratizadores de gobiernos y sociedades. El status de la mujer en la Ciencia Política se desarrolla como parte de un ambiente social, cultural, económico y político más amplio (Agapiou-Josephides, 2001).

En el presente artículo, junto con realizar una descripción analítica de la situación de la mujer en los ámbitos tradicionales de ejercicio disciplinario y de actuación gremial, argumentamos que el panorama politológico chileno y la relativa ausencia de mujeres no se explica sólo por el canon epistemológico de la disciplina, de corte estructural y endógeno, sino por la intervención de otros dos tipos de factores: la dimensión institucional de la disciplina en Chile (asociada principalmente con la transmisión y generación del conocimiento, su base material y los contenidos 
temáticos) y factores contextuales más amplios (relativos al sistema político, en su conjunto). En este sentido, el caso de Chile presentaría similitudes con la situación de la mujer en la Ciencia Política observada en contextos de transición a la democracia (Uriarte, 1997).

Una advertencia: cuando hablamos del posible vínculo entre la categoría mujer y la disciplina de la Ciencia Política, nos encontramos con un fenómeno curioso, y probablemente único, en las Ciencias Sociales: la estrechísima relación entre sujeto y objeto de investigación. Pareciera que fuera imprescindible ser mujer y feminista para investigar en este campo. El desarrollo de los estudios acerca del género y política están, para el caso de la Ciencia Política, vinculados íntimamente al desarrollo del movimiento feminista, al menos, en los países industrializados. A su vez, el discurso feminista se encuentra ligado de manera explícita a dicho movimiento, tanto dentro como fuera de la academia, con consecuencias paradójicas: la marginación al interior de la academia coexiste con un evidente proceso de difusión fuera de las universidades. Sin embargo, también es justo reconocer que no todas las cientistas políticas están vinculadas a las preocupaciones propias del campo de género y política (Ferguson, 1987; APSA, 1992; Uriarte 1997).

Procedemos a organizar nuestra argumentación en cuatro bloques: una primera constatación empírica de la minoritaria situación de la mujer en la disciplina, tal como ella se organiza en Chile; en segundo lugar, una exploración de los problemas epistemológicos que configuran una disciplina poco acogedora para el abordaje y comprensión de las dimensiones relativas al género, particularmente a partir de la experiencia en Estados Unidos; en tercer término, una referencia a la situación de juventud institucional de la Ciencia Política en Chile y una cuarta sección, con una reflexión acerca del impacto de los factores contextuales existentes en Chile, de carácter político-institucional, que sometieron la demanda de participación política de las mujeres a una evidente subordinación al interior de la agenda política de los gobiernos de la Concertación de Partidos por la Democracia, desde el año 1990 a la fecha. Concluimos analizando las condiciones de posibilidad de la interfase entre el género y la Ciencia Política, a la luz del estado actual de la disciplina.

\section{Ejercicio disciplinario: ¿cosa de hombres?}

¿Dónde están las mujeres en el ejercicio disciplinario? Existen diferentes niveles para medir la presencia de las mujeres en la profesión (Sarkees y McGleen, 1992): su presencia en la enseñanza, tanto a nivel de estudiantes como de docentes; en la investigación; y en las publicaciones reconocidas como tradicionales de la Ciencia Política, observando la participación femenina como los contenidos de los artículos. Un 
cuarto nivel de observación se centra en la organización profesional de la disciplina, con un énfasis especial en la instancia gremial de la misma: la Asociación Chilena de Ciencia Política (ACCP), que ha experimentado un funcionamiento ininterrumpido desde el año 1983 a la fecha y que es una de las pocas de la región (junto con la argentina y brasileña) en mantener su afiliación a la Asociación Internacional de Ciencia Política (IPSA). En la ACCP se observa la participación femenina en su ente máximo de decisión, el Directorio, así como el tratamiento de los temas de género en los congresos de Ciencia Política, instancia de reunión bianual de los cientistas políticos chilenos. Las dificultades para reunir la información que se presenta a continuación no han sido menores, producto del desinterés en la reflexión específica sobre estos temas, que se suma a los avatares que la misma ACCP ha debido experimentar, tanto por el contexto sociopolítico en el que nació (en pleno gobierno militar) como por sus dificultades materiales y financieras intrínsecas.

\section{Docencia}

En su análisis del perfil de los profesores de pregrado, Fuentes y Santana (2005) constatan la hegemonía masculina en la disciplina. Si bien ambos autores recogen y mezclan datos de programas de Ciencia Política y de Administración Pública, el resultado final sólo para la Ciencia Política no debiera verse alterado. En su esencia, el estudio de los autores registra el bajo número de mujeres $(24,5 \%)$ que ejercen actividad docente en Ciencia Política. Además, avanzan en señalar que, si bien en Chile los niveles de participación laboral femenina son del orden del $36 \%$, cuando se controla la variable escolaridad, la inserción laboral de la mujer de alta escolaridad es cercana al 50\%, lo que hace más llamativa la cifra encontrada. Por otra parte, al momento del estudio, constatan paralelismos entre dicho porcentaje $(24,5 \%)$ y el promedio de inserción de la mujer en asuntos públicos a nivel nacional, cercano al $20 \%$, según cifras de FLACSO-Chile (2005).

En el cuadro No 1 mostramos como la proporción cercana al 20\% se mantiene, recurriendo a información recogida sólo para el año 2005. En la composición del equipo de gestión de los proyectos de pregrado se observa una participación más paritaria, pero las bajas cantidades de la muestra hacen inevitables ciertos grados de distorsión. La carrera con más presencia de mujeres, al momento del estudio, era la que actualmente ofrece la Universidad Diego Portales, tanto a nivel del cuerpo docente como en su equipo administrativo. Es importante destacar que dicha carrera, en ese momento, era la única dirigida por una mujer, lo que puede ser observado como un ejemplo de que contar con mujeres en la administración de la universidad que compartan la idea de mejorar la situación de la mujer es muy útil para promover su avance numérico. 


\section{Cuadro 1}

Situación de las mujeres a nivel de la docencia y de conformación de equipos directivos, en programas de pregrado en Ciencia Política (año 2005)

\begin{tabular}{lcccccc}
\hline Docencia y gestión & Académicos & mujeres & $\%$ & $\begin{array}{c}\text { Equipo } \\
\text { directivo }\end{array}$ & mujeres & $\%$ \\
\hline Universidad Central & 20 & 4 & 20,0 & 1 & 1 & 100 \\
Universidad de las Américas & 5 & 1 & 20,0 & 2 & 1 & 50 \\
Universidad Arcis & 24 & 5 & 20,8 & 2 & 1 & 50 \\
Universidad Academia de & & & & & & \\
Humanismo Cristiano & 10 & 2 & 20,0 & 1 & 0 & 0 \\
Universidad del Desarrollo & $\mathrm{S} / \mathrm{I}$ & $\mathrm{S} / \mathrm{I}$ & $\mathrm{S} / \mathrm{I}$ & 1 & 0 & 0 \\
Universidad Gabriela Mistral & $\mathrm{S} / \mathrm{I}$ & $\mathrm{S} / \mathrm{I}$ & $\mathrm{S} / \mathrm{I}$ & 2 & 1 & 50 \\
Universidad Diego Portales & 10 & 6 & 60,0 & 3 & 3 & 100 \\
Universidad Católica & 30 & 4 & 13,3 & 3 & 1 & 33,3 \\
\hline
\end{tabular}

Fuente: Elaboración propia, en base a datos proporcionados por los programas de Licenciatura en Ciencia Política.

Con relación a la presencia por sexos, en el estudiantado, vemos una situación distinta. Para el caso de pregrado, la información que se presenta es la disponible a la fecha. En la suma total, se aprecia un virtual empate en presencia por sexos, aunque cuando se analiza por programas de estudio específicos, observamos diferencias. Es importante informar que, para el año 2003, la participación de mujeres en la enseñanza de pregrado, en general en Chile, era del $47 \%$ frente a un $53 \%$ de hombres (El Mercurio, 2005).

Con relación al postgrado, presentamos datos de los programas más asociados a la Ciencia Política, con mayor tradición y permanencia en el tiempo, pertenecientes a la Universidad de Chile y a la Universidad Católica. Llama la atención que la diferencia de participación por sexo en ambos programas es de siete puntos porcentuales $(57 \%$ de hombres frente a $43 \%$ de mujeres). Es posible observar que la participación femenina en estudios de postgrado en Ciencia Política es levemente mayor que el porcentaje de mujeres presentes en estudios de postgrado en Chile, a nivel general $(41 \%)$. Los hombres, mientras tanto, ostentan una presencia del 59\% (El Mercurio, 2005). 


\section{Cuadro 2}

Presencia, por sexos, en la matrícula de pregrado (Licenciatura en Ciencia Política)

\begin{tabular}{|c|c|c|c|c|c|c|}
\hline Universidad & Promoción & $\mathbf{N}^{\circ}$ mujeres & $\% \mathbf{m}$ & $\mathbf{N}^{\circ}$ hombres & $\% \mathbf{h}$ & $N^{0}$ total \\
\hline \multirow[t]{6}{*}{ Diego Portales } & 2002 & 9 & 53 & 8 & 47 & 17 \\
\hline & 2003 & 11 & 45 & 13 & 55 & 24 \\
\hline & 2004 & 15 & 47 & 17 & 53 & 32 \\
\hline & 2005 & 19 & 51 & 18 & 49 & 37 \\
\hline & 2006 & 24 & 31 & 43 & 69 & 77 \\
\hline & Total & 78 & 44 & 99 & 56 & 177 \\
\hline \multicolumn{7}{|l|}{ Academia de } \\
\hline \multirow[t]{6}{*}{ Humanismo Cristiano } & 2002 & 4 & 34 & 8 & 66 & 12 \\
\hline & 2003 & 4 & 66 & 2 & 34 & 6 \\
\hline & 2004 & 9 & 56 & 7 & 44 & 16 \\
\hline & 2005 & 6 & 46 & 7 & 54 & 13 \\
\hline & 2006 & 9 & 27 & 24 & 73 & 33 \\
\hline & Total & 32 & 40 & 48 & 72 & 80 \\
\hline \multirow[t]{13}{*}{ Católica de Chile } & 1995 & 8 & 32 & 17 & 68 & 25 \\
\hline & 1996 & 27 & 56 & 21 & 44 & 48 \\
\hline & 1997 & 46 & 61 & 29 & 39 & 75 \\
\hline & 1998 & 59 & 60 & 40 & 40 & 99 \\
\hline & 1999 & 51 & 54 & 43 & 46 & 94 \\
\hline & 2000 & 65 & 59 & 45 & 41 & 110 \\
\hline & 2001 & - - & & & & \\
\hline & 2002 & 35 & 42 & 47 & 58 & 82 \\
\hline & 2003 & - & & & & \\
\hline & 2004 & - & & & & \\
\hline & 2005 & 33 & 79 & 9 & 21 & 42 \\
\hline & 2006 & - & & & & \\
\hline & Total & 324 & 56 & 251 & 44 & 575 \\
\hline \multirow[t]{4}{*}{ Arcis (Casa Central) } & 2002 & 9 & 45 & 20 & 55 & 29 \\
\hline & 2003 & 18 & 41 & 26 & 59 & 44 \\
\hline & 2004 & 45 & 61 & 22 & 39 & 67 \\
\hline & 2005 & 20 & 34 & 38 & 66 & 58 \\
\hline \multirow[t]{3}{*}{ Arcis (Sede Valparaíso) } & 2003 & 6 & 43 & 8 & 57 & 14 \\
\hline & 2004 & 8 & 53 & 7 & 47 & 15 \\
\hline & 2005 & 12 & 52 & 11 & 48 & 23 \\
\hline \multirow[t]{4}{*}{ Arcis (Sede Magallanes) } & s) 2003 & 6 & 40 & 9 & 60 & 15 \\
\hline & 2004 & 8 & 100 & 0 & 0 & 8 \\
\hline & 2005 & 6 & 26 & 17 & 74 & 23 \\
\hline & Total & 138 & 47 & 158 & 53 & 296 \\
\hline \multirow[t]{5}{*}{ Del Desarrollo } & 2002 & 18 & 69 & 9 & 34 & 26 \\
\hline & 2003 & 15 & 38 & 25 & 62 & 40 \\
\hline & 2004 & 44 & 59 & 32 & 41 & 76 \\
\hline & 2005 & 51 & 69 & 23 & 31 & 74 \\
\hline & Total & 128 & 59 & 89 & 41 & 217 \\
\hline \multirow[t]{2}{*}{ Central } & 2005 & 75 & 45 & 92 & 55 & 167 \\
\hline & Total & 75 & 45 & 92 & 55 & 167 \\
\hline
\end{tabular}


Continuación Cuadro 2

\begin{tabular}{|c|c|c|c|c|c|c|}
\hline Universidad & Promoción & $\mathbf{N}^{\circ}$ mujeres & $\% \mathbf{m}$ & $\mathrm{N}^{\circ}$ hombres & $\% \mathbf{h}$ & $N^{0}$ total \\
\hline \multirow[t]{2}{*}{ De las Américas } & 2005 & 26 & 65 & 14 & 25 & 40 \\
\hline & Total & 26 & 65 & 14 & 35 & 40 \\
\hline \multirow[t]{11}{*}{ Gabriela Mistral } & 1997 & 5 & 63 & 3 & 37 & 8 \\
\hline & 1998 & 7 & 64 & 4 & 36 & 11 \\
\hline & 1999 & 4 & 40 & 6 & 60 & 10 \\
\hline & 2000 & 8 & 47 & 9 & 53 & 17 \\
\hline & 2001 & - & & & & \\
\hline & 2002 & - & & & & \\
\hline & 2003 & - & & & & \\
\hline & 2004 & - & & & & \\
\hline & 2005 & 8 & 10 & 66 & 90 & 74 \\
\hline & Total & 32 & 27 & 88 & 73 & 120 \\
\hline & TOTAL & 832 & 49,8 & 839 & 50,2 & 1.671 \\
\hline
\end{tabular}

Fuente: Elaboración propia, en base a datos del Ministerio de Educación, División de Educación Superior.

\section{Cuadro 3}

Presencia por sexos en los programas de postgrado en Ciencia Política

Alumnos Postgrado Ciencia Política

\begin{tabular}{|c|c|c|c|c|c|c|}
\hline Universidad & Año & $\mathrm{N}^{0}$ mujeres & $\% \mathrm{~m}$ & $\mathrm{~N}^{0}$ hombres & $\% \mathbf{h}$ & $\mathrm{N}^{0}$ total \\
\hline \multirow[t]{14}{*}{ De Chile } & 1993 & 8 & 15 & 46 & 85 & 54 \\
\hline & 1994 & 11 & 17 & 54 & 83 & 65 \\
\hline & 1995 & 22 & 21 & 83 & 69 & 103 \\
\hline & 1996 & & & & & \\
\hline & 1997 & & & & & \\
\hline & 1998 & 18 & 32 & 49 & 68 & 56 \\
\hline & 1999 & 15 & 27 & 41 & 73 & 56 \\
\hline & 2000 & 21 & 33 & 43 & 67 & 64 \\
\hline & 2001 & & & & & \\
\hline & 2002 & & & & & \\
\hline & 2003 & 294 & 56 & 228 & 44 & 522 \\
\hline & 2004 & & & & & \\
\hline & 2005 & 13 & 43 & 17 & 57 & 30 \\
\hline & Total & 402 & 42 & 561 & 58 & 963 \\
\hline \multirow[t]{7}{*}{ Católica de Chile } & 2001 & 20 & 34 & 38 & 46 & 58 \\
\hline & 2002 & 27 & 63 & 16 & 27 & 43 \\
\hline & 2003 & 21 & 44 & 27 & 56 & 48 \\
\hline & 2004 & & & & & \\
\hline & 2005 & 29 & 60 & 19 & 40 & 48 \\
\hline & Total & 97 & 42 & 100 & 58 & 197 \\
\hline & TOTAL & 499 & 43 & 661 & 57 & 1.160 \\
\hline
\end{tabular}

Fuente: Elaboración propia, en base a datos del Ministerio de Educación, División de Educación Superior. 
Otra faceta relevante a analizar en el ámbito de la docencia, para los fines del presente artículo, es aquella relativa a los programas de estudios, identificando la existencia de cursos independientes que aborden distintos aspectos dentro del campo género y política, o bien, de unidades específicas referidas al género dentro de cursos temáticos más amplios. Por ejemplo, existe una tendencia, en la mayoría de las universidades, a incorporar el aporte proveniente de la teoría política feminista dentro del marco más amplio de la Teoría Política contemporánea. Sin embargo, no estamos en condiciones de afirmarlo taxativamente por cuanto no se dieron las facilidades para acceder a los programas de estudio solicitados.

\section{Investigación}

La fuente principal de información en esta materia, en lo que respecta a fondos públicos concursables, es el denominado Fondecyt (Fondo de Desarrollo Científico y Tecnológico). En él, la Ciencia Política se encuentra inserta en el grupo de estudio de Ciencias Jurídicas, desde el año 1990.

Si observamos el período de tiempo comprendido desde el año 1991, en base a los datos disponibles, recién en el año 1998 una mujer gana este fondo concursable, representando en ese momento el $20 \%$ de participación dentro de la disciplina. Los momentos de mayor presencia femenina han sido los años 2003, 2004 y 2005, alcanzando el 50\%, con 1, 2 y 1 proyectos ganados por mujeres, respectivamente. Como saldo final, desde 1991 a la fecha se han ganado 44 proyectos en el campo de la Ciencia Política, con una participación global de mujeres del 18\% (8). Al bajo número de proyectos ganados, ya no sólo por mujeres, sino por cientistas políticos, sin consideración de su sexo, es importante destacar la concentración que se produce en algunas de estas mujeres. De las 8 indicadas, 5 proyectos se han concentrado sólo en dos investigadoras ( 3 y 2 , respectivamente).

Desde el punto de vista de los intereses disciplinarios, ningún proyecto ganador corresponde a los temas propios del campo de estudio género y política.

\section{Organización profesional de la disciplina}

La participación de mujeres en los órganos asociativos gremiales es un indicador relevante, particularmente para el caso de Chile, donde la precariedad institucional de la disciplina ha venido a ser compensada, de alguna manera, por la vigencia de una estructura que permanece desde el año 1983. Creada en el contexto del régimen militar, la Asociación fue en sus inicios un espacio de complicidades políticas, muy vinculadas al interés de recuperación de la democracia, más que académicas y profesionales. Es ésta una función que también cumplieron muchos de los centros académicos independientes durante ese período (Puryear, 1994). Con el correr del tiempo, y 
a pesar de no disponer de la necesaria solvencia económica que le permita autosustentarse (funciona al alero de la institución de adscripción de la persona que detente la presidencia de turno), ha logrado desarrollar actividades gravitantes dentro del contexto disciplinario nacional tales como el Congreso Nacional de Ciencia Política, con carácter bianual. Hasta la fecha se han desarrollado siete congresos nacionales.

Es posible observar que la presencia de cientistas políticas en su directiva, en algunos períodos, es un poco mayor que el promedio de mujeres presentes en el ejercicio docente. Es importante hacer notar, más como curiosidad que como hipótesis explicativa, que cinco de las mujeres que han sido electas para integrar la Directiva son de origen extranjero.

\begin{tabular}{lcccc}
\hline Asociación Chilena de Ciencia Política & Período & Cargos & Mujeres & $\%$ \\
\hline Alicia Frohmann & $1991-1993$ & 7 & 1 & 14,3 \\
Carmen Fariña & $1994-1996$ & 7 & 1 & 14,3 \\
Mercedes Aubá & $1996-1998$ & 7 & 1 & 14,3 \\
Ma. de los Ángeles Fernández y & & & \\
Amparo Menéndez Carrión & $1998-2000$ & 7 & 2 & 28,6 \\
Ma. de los Ángeles Fernández (presidenta) & & & 2 & 28,6 \\
y Jessica Smith & $2000-2002$ & 7 & 1 & 14,3 \\
Olga Ulianova & $2002-2004$ & 7 & 2 & 28,6 \\
Pamela Figueroa y Oriana Piffre & $2004-2006$ & 7 & \\
\hline
\end{tabular}

El género también ha estado presente en los congresos de la disciplina desde muy temprano, aunque ha sido necesario recurrir usualmente a desarrollos teóricos y a exponentes provenientes de otros campos y saberes, e incluso a centros que no tienen necesariamente un carácter académico, dada la ausencia en la Ciencia Política chilena de trabajos e investigaciones significativas en los temas relacionados con el género y la política.

\section{El género en los congresos de la Asociación Chilena de Ciencia Política (ACCP)}

1992: Panel "Políticas Públicas": Los problemas de género en el debate de las políticas públicas. A cargo de CEM (Centro de Estudios de la Mujer).

1996: Panel "Los temas de género y la participación política". A cargo de Carmen Fariña, cientista política.

1999: Panel "Género y poder: discursos, prácticas e instituciones". No hay participación de cientistas políticos. El tema se aborda desde otras disciplinas.

2002: Panel "Género y esfera política". No hay participación de cientistas políticos. El tema se aborda desde otras disciplinas. 
Un tercer nivel de indagación es la membresía a la organización profesional. Dada la carencia de datos confiables y sistematizados referentes a todo el período de funcionamiento de la ACCP, nos remitimos a fotografiar la situación para el año 2006, donde se observa una participación femenina superior al 30\%, en distintos niveles:

- Membresía (miembros plenos, incluye estudiantes):

Hombres: $63 \%$

Mujeres: 37\%

- Participación activa (esperada) en el $8^{\circ}$ Congreso Nacional de Ciencia Política, a realizarse en noviembre del año en curso:

Propuestas de presentación de papers:

Hombres: $64 \%$

Mujeres: $36 \%$

Coordinación de paneles:

Hombres: $67 \%$

Mujeres: $33 \%$

\section{Publicaciones}

Se ha planteado que las publicaciones académicas están fuertemente conectadas con las posibilidades de promoción, posición estable, incrementos salariales y reconocimiento profesional, entre otros aspectos (Mathews y Andersen, 2001). Para el caso de Chile, dichos incentivos serían más nominales que reales, debido a la casi inexistente carrera académica, salvo en las universidades tradicionales (Chile y Católica) y, más recientemente, en la Universidad Diego Portales.

De acuerdo a la experiencia norteamericana, la brecha de género también sería observable en lo que se refiere al ámbito de las publicaciones en Ciencia Política, tanto en revistas académicas como en libros, indicando que es la medida más crítica de involucramiento de mujeres. Si bien se ha ido estrechando paulatinamente, todavía hay una sobrerrepresentación de autorías masculinas, en una relación de dos a uno. Los factores que se indican para explicar esta situación son la ambición, reputación, mérito, apoyo y recursos institucionales, redes profesionales, relaciones de apadrinamiento, tema de investigación y metodología y, por cierto, tiempo disponible ya que son las mujeres las que dilatan o interrumpen sus carreras debido a las obligaciones propias de la crianza de los hijos. La brecha se acrecienta cuando los artículos abordan temas relacionados con género, como categoría analítica, y su relación con la política, salvo en journals especializados como Women and Politcs, produciéndose así un 
fenómeno de "guetización": las mujeres que investigan sobre temas de género y política terminan publicando en journals especializados en asuntos de género. Se ha señalado que una de las cuestiones que incide en las mayores posibilidades de publicación es la construcción de redes, por cuanto la productividad también es el resultado de la posición que se ocupe en el sistema de comunicación en la disciplina. Por último, resulta interesante destacar como fenómeno adicional, que las posibilidades de que los hombres citen las investigaciones de las mujeres son menores (Sarkees y McGlen, 1992; Mathews y Andersen, 2001).

Si bien pudiera resultar azaroso extrapolar las mismas razones para explicar la subrepresentación editorial femenina para el caso de Chile, por la diferencia de contextos, resulta de interés indagar la plausibilidad de las hipótesis esgrimidas para el caso anglosajón, observando la situación chilena. Se muestran, a continuación, los gráficos de la evolución de la participación femenina en las dos revistas más emblemáticas de la Ciencia Política chilena: Política, de la Universidad de Chile, y Revista de Ciencia Política, de la Universidad Católica.

Es posible observar, para ambos casos, un ligero ascenso en los últimos años, aunque la brecha de participación editorial se mantiene entre ambos sexos.

En cuanto a los consejos editoriales de ambas publicaciones, la primera contiene una presencia femenina del $33,3 \%$ (cuatro mujeres y doce hombres), mientras la segunda es de 14,2\% (una mujer y siete hombres).

Una tarea pendiente es la exploración de los temas que abordan las pocas mujeres que han publicado, hasta ahora, en nuestras revistas disciplinarias. En Estados Unidos, las mujeres muestran preocupación por la construcción social de los roles de género, una evaluación crítica de los estereotipos asignados a los deberes profesionales y por el impacto, en la arena política, de las organizaciones de mujeres. Por su parte, los hombres se focalizan en las instituciones formales, teoría política o en la teoría legal (Kelly, Williams y Fisher, 1994).

Como primer balance general, es posible afirmar que la mujer en la Ciencia Política chilena ha estado subrepresentada en términos profesionales con un porcentaje de participación en las actividades de docencia cercano al indicado por Santana y Fuentes (2005). Aunque, y desde la perspectiva del estudiantado, se observa una presencia casi paritaria en la matrícula por género a nivel de pregrado, y una mayor presencia femenina a nivel de postgrado.

Por otra parte, el género y su relación con el poder, como campo de estudio, ha sido abordado generalmente desde otras disciplinas. Tal situación ha sido puesta en evidencia al interior de los congresos organizados por la Asociación Chilena de Ciencia Política, dada la ausencia de investigación nacional en la materia. 


\section{Artículos por género, Política, \\ Universidad de Chile \\ Periodos 1990-2005}

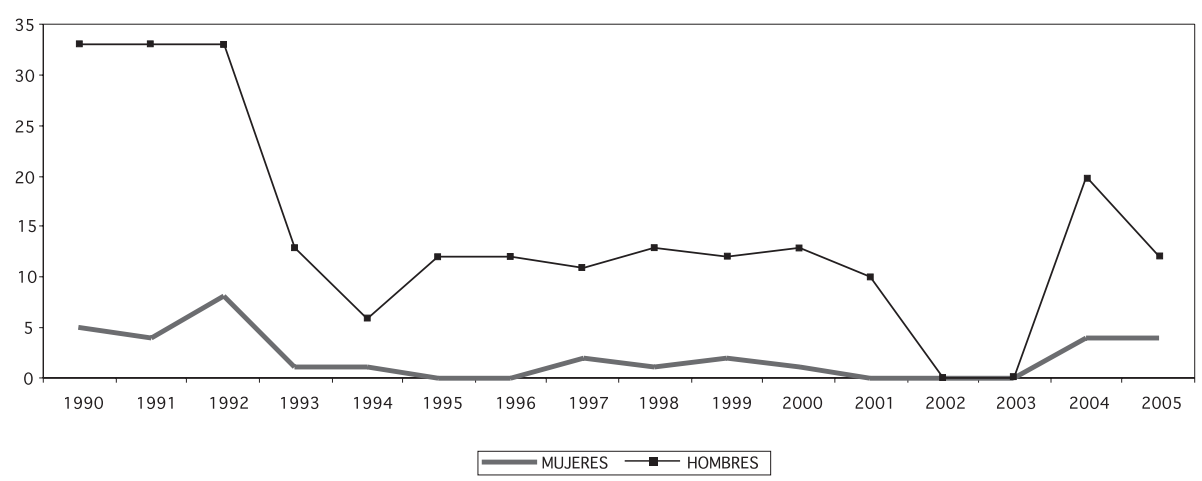

Fuente: Gráfico elaborado con datos recolectados a través de la revisión de ejemplares.

* Durante los años 2002 y 2003 no hubo publicación de revistas.

\section{Artículos por género, Revista de Ciencia Política, Universidad Católica \\ Periodos 1990-2005}

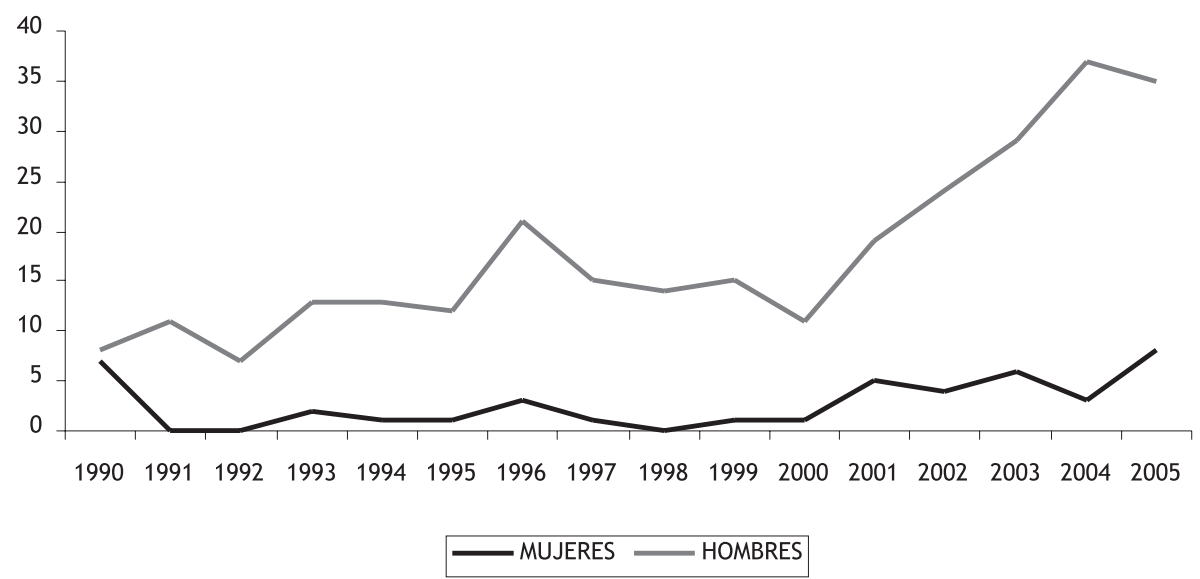

Fuente: Página web de la revista y revisión de sus catálogos.

Finalmente, y en cuanto a la participación femenina en la ACCP, su presencia en la Directiva de dicha asociación ha oscilado, de manera poco consistente, entre el 14 y el $28 \%$. Sin embargo, su presencia en la membresía de la ACCP en el año 2006 y en las distintas instancias del próximo Congreso Nacional de Ciencia Política supera actualmente el 30\%. Esta última cuestión evidencia una situación cuyo desarrollo merece ser 
observado con detalle. Sobre todo teniendo en cuenta que algunos autores han indicado que una masa crítica cercana al 30\% de mujeres es necesaria para crear dinámicas que promuevan decisiones de calidad y que faciliten la valoración de la perspectiva de mujer (Agapiou-Josephides, 2001).

\section{Ciencia Política: ¿ejemplo de misoginia epistemológica?}

El carácter político de las relaciones hombre-mujer y la idea de que "lo personal es político" (la política estaría en todas las decisiones que configuran nuestras vidas, y no sólo en las que se toman en el escenario restringido de lo que se describe convencionalmente como "política") está ampliamente aceptado hoy en la academia. Por otro lado, la forma en que la ley, los medios de comunicación y las personas, en general, se acercan a los temas de género ha cambiado sustancialmente. La experiencia de las mujeres es hoy considerada y constituye un campo de estudio, tanto en la nueva área de "estudios de la mujer" como en las disciplinas tradicionales (Chapman, 1996).

Sin embargo, esto es relativamente nuevo. Por mucho tiempo, las mujeres estuvieron ausentes de los análisis académicos en Ciencias Sociales, con excepción del tema de la familia, desarrollado hasta la década de los setenta, y del abordaje puntual realizado por Duverger, en la década de los cincuenta. La cuestión del género fue generalmente ignorada en la reflexión en las Ciencias Sociales, mientras que, por el contrario, términos genéricos como "trabajador" o "político" eran definidos implícitamente como masculinos.

La reflexión feminista es diversa en temas, teorías y métodos. Si bien existen distintas visiones, convergen en su concepción de las instituciones sociales y los valores culturales como estructuradores de roles y relaciones de género (Vianello y Moore, 2000). Por otra parte, las preocupaciones intelectuales feministas acerca de la mujer y del poder no están limitadas a la Ciencia Política. Los desarrollos acerca del movimiento de mujeres, en teoría feminista, junto con el crecimiento de los estudios interdisciplinarios sobre la mujer, responden a ciertas dimensiones y preocupaciones políticas.

Los estudios feministas sobre mujer y política tienen por objetivo corregir los sesgos previos del mainstream de la Ciencia Política. En efecto, las críticas a la Ciencia Política empírica han mostrado una preocupación especial frente al descuido de los enfoques behavioristas tradicionales sobre la mujer, que han descrito un estereotipo femenino en el ámbito de los roles políticos. Nos referimos, por ejemplo, al comportamiento electoral de las mujeres, visto como conservador, o al supuesto desinterés de las mujeres por la vida política de carácter institucional. Las feministas dise- 
ñaron una agenda de investigación que cambiaría estas apreciaciones, refutando tanto la forma en que la política era practicada como la manera en que era comprendida. Se pensaba que utilizando el concepto de "género" era posible rechazar las definiciones de la política que implicaban la división entre lo público y lo privado y avanzar en el reestudio y examen de las producciones de la Ciencia Política convencional en base a las divisiones de género (Carroll y Zerilli, 1993; Lovenduski, 1998).

Esta vertiente también es abordada por Dietz (2003) cuando plantea que es importante reconocer que el feminismo es un movimiento social y político históricamente constituido, tanto local como global, con un propósito emancipatorio y un contenido normativo. En Ciencias Sociales, el feminismo instala un objeto (mujer), identifica un problema (la sujeción y la objetivación de la mujer a través de las relaciones de género) y expresa varios objetivos (por ejemplo, transformar las relaciones de dominación; terminar con la discriminación sexual; asegurar la liberación sexual femenina; luchar por los intereses y deseos de las mujeres; transformar las estructuras institucionales y legales, "generizar" la democracia) en nombre de principios específicos (tales como igualdad, derechos, libertad, autonomía, dignidad, autorrealización, reconocimiento, respeto y justicia, entre otros).

Si bien existe toda una literatura que da cuenta de su impacto y resultados, y que reexamina el efecto ambivalente de la institucionalización de esta nueva área de estudio, en una perspectiva evaluativa, nos interesa acá colocar el acento en otro aspecto del problema. Las evaluaciones realizadas por las académicas dedicadas a este campo de estudio han llegado a reconocer que, aunque se intentara avanzar en un estudio más sistemático y menos fragmentario del comportamiento político de la mujer, ello no resolvería los problemas básicos encontrados en la relación entre el género y los protocolos de investigación tradicionales de la disciplina. En esta línea, existiría un fenómeno de "asintonía estructural", una suerte de contradicción vital en el proceso de interfase entre la Ciencia Política y el género, asentada en la gestalt misma de la disciplina, que estaría más bien orientada al estudio de variables de carácter dicotómico y a los valores implícitos asociados a ella. Dado que el género se expresa en un campo relacional, y no como una variable dicotómica, y en áreas tales como la representación política, las políticas públicas y las instituciones políticas, la preferencia por los modelos parsimoniosos y elegantes, así como por las hipótesis esbeltas y los datos medibles, son elementos de la Ciencia Política que la harían poco acogedora a los estudios de género. Es por ello que la epistemología disciplinaria y su heurística ofrecen una clara oportunidad para examinar la subrepresentación femenina en este campo (Ghittens, 1983; Lovenduski, 1998).

Bourque y Grossholtz (1998) abundan más en estas apreciaciones cuando denuncian la insistencia de los cultores de la disciplina en identificar datos que analizan e interpretan, basados en una definición estrecha de la 
política que limita la actividad a un conjunto de roles que están en la sociedad, estereotipados como masculinos. Sostienen que las mujeres no podrán nunca aparecer participando plenamente en la actividad política como producto de los presupuestos elaborados acerca de la naturaleza de lo político y las definiciones que de ellos se desprenden. El problema central, a su juicio, es que las mujeres no son diferentes en política a los hombres pero, al mismo tiempo, sí existen diferencias: si los cientistas políticos evitaran los presupuestos con los que emprenden sus investigaciones y encontraran diferencias para indagar más profundamente, encontrarían hipótesis interesantes sobre la vida política. Randall (1991), en términos similares, señala que para crear un espacio dentro de la disciplina que posibilitara la indagación de una agenda feminista, fue necesario cambiar la forma en que la disciplina comprendía su propio objeto de estudio, esto es, lo que se supone que es la política, los aspectos de la política que concitan mayor atención, y los lugares donde la política se sitúa. El autor avanza en señalar que las mujeres somos biológicamente diferentes, a pesar de que las feministas liberales recién estarían comenzando a enfrentar las implicaciones de este reconocimiento. A raíz de este conflicto, surge la preocupación por indagar en qué medida las mujeres serían diferentes así como las consecuencias de esta posible diferencia.

¿Cuál es la situación actual? A nivel de la disciplina de la Ciencia Política como un todo, no ha habido cambios significativos que desplacen la hegemonía de la epistemología positivista, que trabaja problemas políticos convencionales y con metodologías esencialmente cuantitativas y derivadas del Rational Choice (APSA 2001). Partiendo por el estudio de la mujer y la política como un asunto convencional, institucionalmente focalizado, con definiciones estado-céntricas de lo que es político, el análisis se ha ido extendiendo a otros ámbitos de lo que puede ser considerado como político. Desde una concepción esencializada de la mujer se transita hacia una búsqueda teórica y metodológica que informe la manera en que las construcciones de dominación y subordinación, que no aparecen vinculadas de manera específica al género, refuerzan, interactúan y determinan el poder político de las mujeres, así como la práctica política. Asistimos hoy a un pasaje desde una percepción inicial del género, como sinónimo de sexo, hacia una intuición epistemológica de que el género está íntimamente relacionado con interacciones dinámicas culturales y específicas. En la actualidad, se propone analizar el género como categoría y como proceso, en la perspectiva de mapearlo completa y cuidadosamente en contextos específicos. Posicionarse desde esta perspectiva lleva al reconocimiento de que las femineidades y las masculinidades son plurales, específicas en sus prácticas y contenido, y con diferentes implicaciones políticas (Mazur, 2004; Beckwith, 2005). Hoy día asistimos a un movimiento: desde "mujer en política" hacia "género y política". Ello implicaría estudiar tanto las interacciones de hombres y mujeres como actores institucionalmente generizados, actuando dentro 
de instituciones generizadas, así como los fenómenos producidos por el ingreso de las mujeres en los espacios tradicionalmente masculinos. La siguiente etapa, por tanto, correspondería al abordaje del género como un concepto relacional complejo y una ideología estructurante, más que como una categoría. Sin embargo, en la Ciencia Política, esta aproximación se encuentra todavía en sus inicios y ello se debería, en parte, a la brecha entre teorías sofisticadas de género y la ausencia de trabajos susceptibles de ser operacionalizados para la investigación científica. En esta etapa, las preocupaciones están básicamente centradas en los enfoques y los métodos, la conceptualización de lo político y la naturaleza "generizada" de las instituciones y procesos políticos (Shils y Krook, 2006; Mackay, 2004). Esa es la tarea que se presenta por delante.

\section{El contexto disciplinario y las posibilidades para el género: ¿un hori- zonte cercano?}

La Ciencia Política en Chile, como modo de indagación y campo intelectual, es relativamente nueva. Ello no quiere decir que no haya existido una tradición previa de estudios y análisis políticos, con escasa diferenciación disciplinaria entre sí. Sin embargo, nos referimos aquí a una forma de discurso y a una práctica académica institucionalizada y formalizada dotada de estructura material, financiamiento, masas críticas vinculadas y ciertas dosis de visibilidad. En este sentido, la Ciencia Política chilena como empresa intelectual relativamente autónoma y en un sentido disciplinario moderno (de acuerdo al patrón norteamericano), hunde sus raíces a fines de los años sesenta, recibiendo el impacto del golpe de Estado de 1973 cuando recién estaba iniciando su despegue. No sólo existe un debate, a nivel internacional, acerca de los dispares efectos de los contextos políticos autoritarios en el desarrollo de la Ciencia Política (Easton et al., 1995), sino que tampoco existe un relativo acuerdo en nuestro país acerca de los efectos del régimen militar: para algunos, el ambiente previo de polarización ideológica y de posterior represión política produjeron, en su conjunto, una plétora de estudios y análisis políticos de gran calidad, al alero de los llamados centros académicos independientes (CAI), los cuales, sin embargo, no alcanzaron a constituir una disciplina (Lechner, 1990). Para otros, durante los años 80 y al inicio de la transición democrática, se habría constituido una infraestructura institucional capaz de generar un desarrollo disciplinario sostenido. Demostrativo de ello serían los programas de estudio, de postgrado y pregrado, así como la cantidad de estudiantes interesados en seguir esta línea de estudios (Rehren y Fernández, 2005).

Independientemente de la ausencia de un consenso entre los cientistas políticos chilenos acerca de la situación en que se encontraba la disciplina a inicios de la transición a la democracia, lo cierto es que si habían 
condiciones materiales esenciales y un cierto despliegue, éste se ha circunscrito a los ámbitos tradicionales de la disciplina (instituciones y procesos políticos, teoría política, relaciones internacionales y políticas públicas).

El sistema democrático no ha representado, para la disciplina, el mejor de los mundos posibles. Se ha llegado a afirmar que la estructura fragmentada del sistema de educación superior chileno, el desaparecimiento de los fondos de fundaciones extranjeras y el precario apoyo material al desarrollo de las Ciencias Sociales ha derivado en una disciplina más débilmente direccionada en la fase postautoritaria (Easton et al., 1995). En este contexto, resalta la explosión de proyectos docentes de pregrado en el campo de la Ciencia Política, lo que a juicio de Fuentes y Santana (2005) responde esencialmente a la lógica de mercado inserta en el sistema de educación superior, de carácter desregulado en la apertura de carreras, entre otros factores.

Este proceso ha llevado a la expansión de los espacios de ejercicio para la docencia, que parecieran ir acompañados de precariedad en los estímulos materiales, rotación y discontinuidad. Es éste un tema al que debe prestarse atención, dadas las imposibilidades de una mayor intencionalidad. Se debería también analizar cómo afecta a las mujeres esta condición, dada su evidente subrepresentación disciplinaria.

Con relación al porcentaje de alumnos por sexo, según los datos suministrados para el año 2005, se observa una notable participación de las mujeres en algunos programas de Licenciatura, llegando en algunos casos al 57\%. La posibilidad de que este porcentaje se refleje en el ejercicio profesional dependerá de un cúmulo de factores que bien pudieran ser los mismos que se diagnostican para el caso europeo, referidos a la carrera académica en sentido específico y que afectan por igual a hombres y a mujeres, o referidos a factores de tipo diferencial entre ambos sexos. Para el primer caso, se habla de las consideraciones financieras para proseguir estudios, falta de información y de autoconfianza, y el deseo de tener un impacto en el mundo real (motivación que no siempre se vincula con la vocación académica).

Con relación al segundo tipo de factores, más relacionados con la situación de la mujer en el mercado de trabajo así como a la reconciliación entre vida profesional y vida familiar, se señalan más específicamente: los estereotipos, los modelos de rol, los compromisos familiares y las limitaciones del tiempo (Akhtar et al., 2005; Agapiou-Josephides, 2001). Para el caso de Chile, existe la agravante de que la tasa de participación laboral femenina es $35 \%$ (versus un $45 \%$ en los países desarrollados), lo que denota problemas estructurales serios para una mayor participación de la mujer en el mercado laboral. Se ha señalado que esta incorporación al mercado de trabajo sería dicotómica: mientas las mujeres más pobres tienen empleos más informales o se desempeñan en el 
servicio doméstico, las de mayor nivel educacional poseen empleos similares a los de las mujeres europeas, pero con una menor tasa de participación. En Chile, el 50\% del total de profesionales son mujeres, similar a la media de los países desarrollados, pero el porcentaje de mujeres directivas y altas funcionarias llega sólo al 24\%, en comparación con los hombres (Schkolnik, 2004), cifra que coincide con el porcentaje de mujeres docentes en nuestra disciplina, según el estudio de Fuentes y Santana (2005).

Es evidente que asistimos a una explosión de programas, tanto a nivel de pregrado como de postgrado, así como a un creciente incremento del número de cultores vinculados a la disciplina. Si ello se refleja en un mayor desarrollo, es un tema debatible por cuanto carecemos de indicadores fidedignos que establezcan la calidad. Altman (2005) informa que tanto la academia norteamericana como la europea han avanzado en el establecimiento de criterios en esa dirección.

Lo que sí es indudable es que, desde una perspectiva temporal, el itinerario de la disciplina en Chile es todavía muy reciente y ello podría explicar de manera parcial la inexistencia de la instalación de áreas que aparecen bien consolidadas en los contextos disciplinarios de otras latitudes. Sin embargo, cabe preguntarse: ¿es la relativa inmadurez institucional el factor que podría explicar el desbalance de género en la Ciencia Política, como ámbito de estudio y en cuanto a la participación profesional de las mujeres? No parece una respuesta del todo plausible, por cuanto otras disciplinas de trayectoria tan joven como la Ciencia Política se han hecho cargo, para el caso chileno, de reflexionar sobre la relación entre el género y el poder, muchas veces en perspectiva interdisciplinaria. Ríos Tobar et al. (2003) brindan una sugerente explicación de los factores que facilitaron su surgimiento en el período postransición y posible vinculación con el movimiento feminista. Nos referimos a la sociología, la antropología, la literatura, la lingüística, entre otros saberes. A continuación, se muestra un cuadro de situación de dichos programas, al año 2005, que da cuenta de la ausencia de cientistas políticos en su interior:

Programas universitarios de género (vigentes al año 2005)

Universidad Austral: ha realizado seminarios y conferencias sobre el tema, a cargo de la Facultad de Medicina.

Universidad de Playa Ancha: imparte el Magíster Interdisciplinario (Historia, Filosofía, Literatura y Lingüística). No hay cientistas políticos en el equipo. En la Facultad de Humanidades se plantea que una de las líneas de investigación es el tema género.

Universidad Academia de Humanismo Cristiano: existe el Programa Pro-género, con la colaboración de la Fundación Instituto de la Mujer, FLACSO y el Centro de Estudios de 
la Mujer (CEM). No hay politólogas en el equipo. La mayoría son sociólogas, psicólogas y antropólogas.

Universidad de Santiago - IDEA: hay algunos proyectos de investigación en relación al tema género, pero no hay cursos especializados en el tema.

Universidad de Chile: en la Facultad de Filosofía y Humanidades hay un Centro de Estudios de Género y Cultura en América Latina, con su correspondiente Magíster. En la Facultad de Ciencias Sociales se ubica el Magíster denominado "Género y Cultura".

Universidad de La Serena: hay un Centro Interdisciplinario de Estudios Latinoamericanos (CIEL), donde imparten un magíster en el mismo tema. No hay participación de cientistas políticos.

Este catastro es corroborado por un estudio reciente que lleva por título "Incorporación del género en la enseñanza universitaria", con fecha 2006, de la Facultad Latinoamericana de Ciencias Sociales (FLACSO) y el Colegio de México. En dicho estudio, la Ciencia Política no aparece vinculada a ninguna iniciativa de postgrado, en relación al género y la sexualidad.

Junto con la juventud de la disciplina, producto de su particular forma de desarrollo en nuestro país y de los efectos ambivalentes del régimen militar, se podría consignar la indiferencia y/o el rechazo de muchos investigadores a ocuparse de esta área de estudio.

Shilds y Krook (2006) y Uriarte (1997) informan de las situaciones que se experimentan, para el caso británico y español. En el primero, se indica que el escepticismo con relación a los valores de la investigación feminista en Ciencia Política se deriva de una utilización favorita de métodos cualitativos (en desmedro de los análisis cuantitativos, privilegiados por los journals), y del cuestionamiento de la objetividad de dichos estudios. Para el segundo, se señala que las connotaciones negativas vienen dadas por dos factores: el primero, porque todo tema relacionado con la mujer ocupa automáticamente un lugar secundario (y no sólo en el mundo académico) y, en segundo término, el efecto negativo de la ideología feminista que está a la base de dichas iniciativas, como correlato de la imagen negativa que el feminismo tiene en muchos sectores de la sociedad.

Será necesario emprender análisis empíricos que permitan probar que estas mismas conjeturas se reproducen para el caso chileno. Asimismo, otro ámbito interesante de exploración es la relativa ausencia de mujeres en las disciplinas de las Ciencias Sociales y las Humanidades, a través de series de tiempo, distinguiendo períodos de "juventud" disciplinaria y de "madurez" (Akhtar et al., 2005). 


\section{Mujeres, política y Ciencia Política: un encuentro que estaba pendiente}

La competencia electoral por la presidencia del cuarto gobierno de la Concertación, durante fines del año 2004 y principios del año 2005, se tornó especialmente atractiva e igualmente tensa: dos mujeres, Michelle Bachelet y Soledad Alvear, pertenecientes a la misma coalición, pero de partidos políticos y mundos ideológicos distintos, concentraban el favoritismo ciudadano. Cuando todavía no se dirimía quién encabezaría la contienda frente a los partidos de la derecha chilena, la que debería zanjarse en elecciones primarias, ya múltiples interrogantes saltaban a la palestra, tales como si las mujeres votarían por una mujer, si el sexo es importante a la hora de orientar la dirección del voto, si la capacidad de liderazgo político está influenciada por el sexo, y hasta si las mujeres hacen la diferencia cuando entran a la arena política, por nombrar sólo algunas. Todas estas interrogantes, que afloraron en el debate público, encontraron a la Ciencia Política chilena sumida en la perplejidad. Sólo cabía recurrir a los estudios sobre género y política realizados en otras latitudes, usualmente democracias occidentales anglosajonas, o bien recoger las respuestas entregadas desde otras orillas disciplinarias, tales como la Sociología o la Historia, que habían acogido la reflexión acerca del género más tempranamente.

La Ciencia Política, tal como se venía desarrollando en nuestro país, se encontraba desvalida de herramientas teóricas y metodológicas para dar respuestas, no sólo eficientes, sino oportunas. Nuestra disciplina, frente a la emergencia del fenómeno político que suponía que una mujer pudiera acceder a la presidencia del país, mostró en dicha contingencia una cierta dosis de irrelevancia.

La realidad entregaba algunas explicaciones sobre la miopía disciplinaria en este ámbito: para el caso de las mujeres, la transición democrática no significó mayores cambios en la posición subordinada que ocupan en la vida política (La Morada, 2004; Humanas, 2005). Distintos indicadores reportan esta situación: Chile es uno de los pocos países de la región que no dispone de una Ley de Cuotas u otras medidas de acción afirmativa y ostenta el lugar duodécimo, de dieciocho países, en relación a la participación parlamentaria femenina, así como el lugar número $71^{\circ}$ a nivel mundial. Hasta la llegada de la presidenta Bachelet al poder, la presencia de las mujeres en el gabinete había alcanzado su nivel más alto durante el gobierno del presidente Ricardo Lagos, con un 29,2\%, porcentaje que disminuyó antes de terminar su período de gobierno. En los otros niveles de la administración pública se observan altibajos durante todo el período postautoritario, lo que se podría explicar por la ausencia de cambios legales e institucionales que permitan asegurar la presencia femenina en el tiempo.

Estos datos evidencian la deuda del proceso de transición a la democracia con la participación política de las mujeres, particularmente en rela- 
ción a su inserción en las instituciones políticas. Se ha tratado de explicar esta situación. Una primera línea argumental dirige la atención hacia el movimiento feminista, el que, si bien realizó una contribución sustantiva a la democratización de las sociedades y a la generación de nuevas institucionalidades responsables de la formulación de políticas públicas con equidad de género, no le asignó la misma prioridad a la importancia que tienen las desigualdades de género en los procesos de gobernabilidad que aspiran a la consolidación democrática (Guzmán, 2003). Otros estudios se dirigen a la explicación institucional, señalando que los contextos de transición a la democracia presentan evidentes restricciones para avanzar en las preocupaciones de género, máxime cuando las parlamentarias no tienen la confianza ni la preocupación suficiente para articular políticas de este tipo (Galligan y Tremblay, 2005). Para el caso de Chile, dicho argumento se circunscribiría a la política de coalición y a cómo la Democracia Cristiana ha logrado el control de la principal institución pública dedicada a estos temas, el Servicio Nacional de la Mujer (Sernam), movilizando un discurso moderado, centrado en el rol de la mujer como madre, dentro del contexto de la familia (Baldéz, 1999). Sin embargo, otra línea argumental observa que dicho organismo constituye un gran logro del movimiento feminista, a pesar de las restricciones propias del período de transición a la democracia (Waylen, 2000). Incluso, se lo observa como un instrumento que no sólo ha alterado el contexto institucional en el cual actúa el movimiento de mujeres, sino que también ha provisto de importantes recursos durante este período, con especial acento en un discurso sobre la igualdad de oportunidades para las mujeres y un conjunto de objetivos en torno a los cuales movilizarse (Franceschet, 2003).

La actual situación, con una mujer Presidenta, gobierno paritario y altas expectativas de las mujeres para que cambie su actual situación, según reportan estudios realizados recientemente (Mori, 2006), debería interpelar a la Ciencia Política local.

En esta línea, Lovenduski señala los ámbitos en los cuales la Ciencia Política podría verse impactada por los aportes procedentes del estudio feminista de la política (Ferguson, 1987). Resulta relevante no sólo indicarlos, sino explorarlos, de acuerdo al estado actual de la disciplina, en nuestro país:

- Una condición disciplinaria importante es el nivel de apertura hacia perspectivas disciplinarias distintas y a debates de tipo epistemológico. Con relación a este punto, Santana y Fuentes (2005) observan en su indagación un conjunto de tendencias que no son sólo de carácter teórico y metodológico, advirtiendo la posible instalación de un clima analítico de tipo ecléctico. Ello posibilitaría un campo de cultivo inicialmente favorable para la instalación de estudios sobre género y política. Esta condición de apertura no es menor, habida cuenta de las 
dificultades detectadas en otras comunidades disciplinarias por las investigadoras que desean dedicarse a este campo. Muchas veces, para desarrollar la reflexión en dichos temas, el costo ha sido la adscripción al mainstream dominante, adoptando su consiguiente metodología de investigación (Ferguson, 1987; Kenney, 1995; Uriarte y Elizondo, 1997). Este camino correspondería a las denominadas académicas "acomodacionistas", que adoptan la epistemología convencional de la disciplina (positivismo, con la explicación científica y la predicción como objetivo y abordaje de temas tales como partidos, voto, Estado-nación, paz y guerra, actitudes políticas y participación, además del uso de metodologías cuantitativas) y añaden a las mujeres como actores políticos. No es éste, sin embargo, el único camino posible para aquellas/os que desean dedicarse a la investigación disciplinaria en el campo del género mediante la utilización de metodologías más sensibles: existe la vía denominada "transformacionista", que adhiere a una epistemología postpositivista (empírica, con la interpretación como objetivo), a nuevos conceptos y a una expansión de los métodos de investigación (etnografía, postestructuralismo, cualitativa, observación participante y entrevista en profundidad) (APSA, 2001).

- La necesidad de prestar atención a los posibles roles cambiantes de las mujeres en el gobierno es un segundo factor determinante. El cultivo de la disciplina a nivel nacional no podrá permanecer ajeno a los debates surgidos durante el período electoral y que, seguramente, se acentuarán con la presencia de mujeres en el equipo de gobierno, la discusión de la reforma electoral, la definición de listas electorales en las próximas elecciones municipales del año 2008, los efectos y diferencias del liderazgo femenino y la dinámica interna de la vida partidista frente a estos procesos, por indicar algunos terrenos de debate. Las posibilidades de desarrollo de la Ciencia Política a nivel nacional dependerá no sólo de su capacidad de reacción frente a las interrogantes que surgirán, sino también de las formas que asumirá dicha respuesta, en el sentido de superar las limitaciones que la Ciencia Política ha evidenciado, avanzando más bien hacia una dimensión menos estrecha de aquello que se considera político, y conjugando protocolos de investigación más diversos y sensibles a la realidad del género. El contexto sociopolítico, para caminar en esta dirección, no pudiera ser más favorable que el actual.

- El impacto de las mujeres y de las feministas organizadas al interior de la Ciencia Política. Ya hemos constatado que al interior de la Ciencia Política no se han constituido programas de estudio centrados en la temática del género, sino que los que existen han sido alojados por otras disciplinas académicas, con particular énfasis en un enfoque interdisciplinario. 
Lo que pudiera hacerse en Chile bien pudiera tener como referencia el camino seguido por las feministas al interior de la disciplina en otros países. ¿Tenderá a configurarse como "gueto", tal como ha sucedido en los países industrializados? La configuración académica e institucional que se adopte permanece incierta debido al escaso número de cientistas políticas que se encuentran impulsando investigaciones en temas relacionados con género y política. Este pequeño número, sin embargo, podría lograr un pronto impacto si es potenciado por las redes académicas transnacionales, habida cuenta de la atención que ha despertado la experiencia política paritaria en curso.

En el ámbito del ejercicio profesional, Santana y Fuentes (2005) recomiendan la utilización de medidas de acción afirmativa a fin de impulsar una mayor presencia de las mujeres en la disciplina. La aplicación de esta medida merece cierto escepticismo si es que tomamos en serio los debates que se han impulsado recientemente en el país en relación a la aplicación de una ley de cuotas para posibilitar una mayor presencia de mujeres en el Congreso. No es tanto la fortaleza de los argumentos de sus detractores, como su control hegemónico de los espacios de debate público en que éstos circulan, lo que hace que exista, al menos a nivel de la elite política y de sectores profesionales informados, un rechazo consistente a este tipo de iniciativas, que tan eficientemente han funcionado en otros países de la región. El caso de Chile viene a ilustrar cómo el individualismo liberal se ha asentado de tal manera que no se perciben oportunidades para el impulso de medidas de acción afirmativa que erosionen la idea de igualdad formal y que sean sensibles a las desigualdades producidas por características de tipo adscriptivo (como el sexo), firmemente instaladas en la cultura jurídica y en la Constitución que ordena la vida del país. Si bien esta última ha sido objeto de reformas sucesivas durante los años de transición, conserva su esencia de origen en el liberalismo clásico, con un énfasis en el reconocimiento de los derechos individuales y un apego a una visión de los derechos que demandan del Estado una omisión, pero ninguna obligación o acción positiva (Claro, 2005). En este contexto, surge como tema a explorar la forma, la naturaleza y las dinámicas en que la ideología liberal dominante en Chile sirve de soporte y refuerzo, no sólo de los discursos y dinámicas políticas, sino de una forma determinada de validación del conocimiento y los estándares exigidos para los procesos de investigación, para el caso de la Ciencia Política, y cómo ello influye en la mayor rapidez o lentitud para el desarrollo de los estudios en el campo del género y los fenómenos de poder.

\section{Conclusiones}

Para entender la situación de la mujer en la Ciencia Política, tal como se ha desarrollado en Chile, tanto desde un punto de vista científico como 
en su expresión más profesional, es necesario tener a la vista, no sólo la forma particular en que el sistema de conocimiento disciplinario entiende la generación de nuevos hallazgos y su validez, sino también las condiciones de historicidad del campo intelectual y los factores sociopolíticos más amplios. En este caso, resulta pertinente tener presente el contexto de la transición política y la forma en que los arreglos institucionales y los actores, tanto políticos como sociales, contribuyeron a diseñar estructuras, procedimientos y campos en los cuales las problemáticas que atañen a la mujer se han abordado de una forma tal, que su expresión más política ha quedado postergada.

¿Cuáles son las condiciones de desarrollo del género como campo de estudio y de reflexión? ¿Cuáles son las posibilidades de desarrollo profesional para las mujeres en el contexto disciplinario? Observamos los primeros esfuerzos, tales como la instalación de programas de investigación como el denominado "Gobernabilidad democrática y representación política en Chile: reforma electoral y ley de cuotas", bajo el alero de la Facultad Latinoamericana de Ciencias Sociales (FLACSO), o el presente número especial dedicado a la problemática del género y la política. Por otra parte, dos cientistas políticas integraron la reciente comisión nombrada por el gobierno de la presidenta Bachelet para elaborar propuestas de reforma al sistema electoral binominal, durante el primer semestre del año 2006.

Desde el punto de vista del clima intelectual y de los enfoques y dilemas de la profesionalización, las señales emitidas en la reciente aprobación de proyectos concursables a fondos públicos (como es Fondecyt) no entregan razones para estar optimistas acerca de la posibilidad de cultivar la Ciencia Política, más allá de los estrechos horizontes definidos por el mainstream dominante, de corte positivista. Esta aprensión se alimenta con el paulatino incremento de la instalación, en Chile, de académicos con formación estadounidense (Fuentes y Santana, 2005). Estos fenómenos llevan a sospechar que existen ciertos límites establecidos por lo que se considera "aceptable" disciplinariamente hoy en Chile, basados en compromisos epistemológicos serios con el positivismo, aunque aparezcan escudados en una aparente neutralidad y en el estímulo a la meritocracia. Altman (2005) señala que, para que ésta exista, deben existir criterios claros y definidos de cómo ponderar la productividad de la investigación y la docencia. ¿Y qué sucede cuando los criterios para ponderación son establecidos por quienes consideran que la generación de conocimiento al interior de la Ciencia Política y los criterios de validez vienen determinados por estándares positivistas? Para Ferguson (1987), dado que el behaviorismo establece una conexión implícita entre los presupuestos metateóricos y la diferencia de género, si el feminismo logra instalarse en la disciplina, sería a costa de su disciplinamiento dentro de los cauces señalados por la corriente dominante. Si ello llega a suceder de esta forma, las posibilidades de acogida y recepción de las preguntas 
y dilemas que experimentan en la actualidad los estudios acerca del género, al interior de la Ciencia Política en las comunidades disciplinarias que ya han avanzado varias fases de dichos estudios, la utilización del género como categoría analítica, la apropiación de metodologías más plurales así como el cruce fertilizante con otras disciplinas (tales como la psicología, la historia y la sociología) (Burns, 2002; Hawkesworth, 2005) estarían claramente limitadas, cuando no coartadas. Shilds y Krook (2006) informan de un excelente ejemplo de estos dilemas en el ámbito específico de la representación política de las mujeres y la respuesta a la pregunta de si las parlamentarias favorecen los intereses propios de las mujeres. Señalan que el conocimiento en este tema, originado en base a números (cantidad de mujeres en los Parlamentos y cantidad de mociones o proyectos presentados), poco añade si no se complementa en términos de conocer lo que sucede con el comportamiento en instituciones y contextos generizados. Por ello, abogan por un pluralismo metodológico que permita incorporar los estudios de caso y a micronivel, como los estudios de datos agregados.

Las aprensiones anteriormente expuestas no significan, en ningún momento, que no deban utilizarse metodologías empíricas. Todo lo contrario: el abordaje de las relaciones entre el género y la política constituye una oportunidad para incorporar otras perspectivas, responder a las preguntas que flotarán en el ambiente (particularmente durante el actual gobierno) y promover un concepto más amplio de lo que se entiende por Ciencia Política.

En el campo de la docencia, deberían ser integrados prontamente cursos y programas de estudio que aborden el estudio del género, desde la perspectiva del objeto de estudio como de metodologías sensibles a su análisis, como parte integral de cualquier programa de estudio en Ciencia Política. La ausencia de este tipo de cursos tendería a revelar no sólo la ignorancia acerca de la importancia de este campo al interior de la disciplina, sino también la insuficiencia del entrenamiento a la que estarían sometidos los estudiantes de la misma (APSA, 2001).

Con relación a facilitar una mayor presencia de las mujeres en todos los niveles, tanto en docencia como en investigación, así como en las instancias de toma de decisiones disciplinaria, se observan dificultades para el impulso de medidas de acción afirmativa, a excepción de contextos institucionales de carácter internacional. Así como en muchos países la acción afirmativa ha sido un eficiente mecanismo de política pública capaz de superar la fragmentación social y la discriminación cultural, en Chile ha tenido pocos espacios, básicamente porque la cultura jurídica, la Constitución vigente y la ideología dominante son esencialmente liberales, con un privilegio desmedido del valor de la igualdad formal y una incomprensión evidente al tipo de situación que viven las mujeres, para quienes la desigualdad se hace patente en ámbitos donde se expresa la 
subjetividad y la intimidad. Ello no debería ser un obstáculo insalvable frente al compromiso de eliminar las desigualdades de género al interior de la disciplina, impulsando formas más flexibles de trabajo y maneras no tradicionales de quehacer profesional que posibiliten una mayor presencia de mujeres. Particular atención debería colocarse en los incentivos salariales. Si bien carecemos de información específica en relación a los niveles de remuneración en la disciplina, está plenamente identificada la brecha salarial existente entre hombres y mujeres: desde 1990 a la fecha, se mantiene una diferencia de $27,5 \%$ a favor de los hombres (Fuentes et al., 2005).

La Ciencia Política en Chile no podrá eludir los dilemas que surgen, buscando un punto de equilibrio con las demandas que se desprenden de su proceso de incipiente profesionalización, con particular acento en los estándares de calidad. Está demostrada la existencia de una relación inversamente proporcional entre una disciplina altamente profesionalizada, con su consiguiente tendencia a la especialización, y su menor influencia en el contexto social más amplio, con cierta discapacidad para hablar acerca de "los grandes temas de nuestro tiempo" (Newton y Vallés, 1990). Si se enfatiza excesivamente la exigencia profesionalizante, bajo el prisma de la hegemonía del mainstream positivista y un academicismo compulsivo, la Ciencia Política no sólo descuidará las exigencias del entorno y sus posibilidades de contribución al debate en curso, sino que hipotecará sus posibilidades de integrar las nuevas tendencias teóricas y metodológicas que emergen en la actualidad, para un análisis comprensivo del género.

\section{Bibliografía}

Agapiou-Josephides, Kalliope. 2001. Political Science. Project Athena: women in the profession. Assession the gender gap in the European Political Science. A preliminary report, Krakow Meeting, Japiellonian University, 17 y 18 de mayo 2001.

Altman, David. 2005. "La institucionalización de la Ciencia Política en Chile y América Latina: una mirada desde el Sur". Revista de Ciencia Política 25 (1): 3-15.

American Political Science Association (APSA). 1992."Improving the status of women in Political Science: a report with recommendations", PS: Political Science and Politics 25 (3): 547-554.

American Political Science Association (APSA). 2001. "The status of women in Political Science: female participation in the professoriate and the study of women and Political Science in the discipline", PS: Political Science and Politics, junio.

Baldéz, Lisa. 1999. "La política partidista y los límites del feminismo de Estado en Chile". En Paul Drake e Iván Jaksic (eds.), El modelo chileno: democracia y desarrollo en los 90. Santiago: Lom Ediciones, 407-433.

Beckwith, Karen. 2005 “A common language of gender?". Politics \& Gender 1 (1): 128-137. 
Bourque, Susan; Grossholtz, Jean. 1998. "Politics an unnatural practice: Political Science looks at female participation". En Anna Phillips (ed.), Feminism and politics. New York: Oxford University Press, 23-43.

Burns, Nancy. 2002. "Gender: public opinion and political action". En Ira Katznelson y Helen V. Milner (eds.), Political Science: the state of the discipline. Washington: W.W. Norton \& Company / APSA, 462-487.

Carroll, Susan J.; Zerilli, Linda. 1993. "Feminist challenges to Political Science". En Ada W. Finifter (ed), Political Science: the state of the discipline. Washington D.C, APSA, 55-76.

Chapman, J. 1997. "La perspectiva feminista". En D. Marsh y G. Stocker (eds.), Teoría y métodos de la Ciencia Política. Madrid: Alianza Editorial, 103-123.

Childs, Sarah; Krook, Mona Lena. 2006. "Gender and politics: the state of the art". Politics 26 (1): 18-28.

Claro, Magdalena. 2005. Acción afirmativa en democracias inclusivas. Santiago: Fundación Equitas.

Corporación Humanas. 2005. Informe regional de derechos humanos y justicia de género 2001-2004. Santiago: Corporación Humanas.

Corporación La Morada. 2004. Mujeres sujeto, mujeres sujetadas. Informe sobre la situación de los derechos humanos de las mujeres en Chile. Derechos civiles y políticos. Santiago de Chile: Corporación La Morada.

Dietz, Mary G. 2003. "Current controversies in feminist theory". Annual Review of Political Science 6: 399-431.

Easton, David et al. 1995. "Introduction: democracy as a regime type and the development of Political Science". En David Easton et al. (eds.), Regime and discipline: democracy and the development of Political Science. Ann Arbor, The University of Michigan Press, 1-23.

Ferguson, Kathy E. 1987. "Male-ordered politics: feminism and Political Science". En Terence Ball (ed.), Idioms of inquiry: critique and renewal in Political Science. Albany: State University of New York Press, 209-229.

Fernández, María de los Ángeles. 2004. “Una disciplina en busca de la profesión". Política 42: 9-12.

2005. "Ciencia Política en Chile: un espejo intelectual". Revista de Ciencia Política 25 (1): 56-75.

Facultad Latinoamericana de Ciencias Sociales (FLACSO). 2005. Agenda democrática, Santiago: Facultad Latinoamericana de Ciencias Sociales.

Franceschet, Susan. 2003. "'State feminism' and women's movements: the impacts of Chile's Servicio Nacional de la Mujer on women's activism". Latin American Research Review 38 (1): 9-32.

Fuentes, Claudio; Santana, Graciela. 2005. “El 'boom' de la Ciencia Política en Chile: escuelas, mercados y tendencias". Revista de Ciencia Política 25 (1): 16-39.

Fuentes, Jeannette, et al. 2005. “Discriminación salarial por género en Chile: una mirada global". Estudios de Economía 32 (2): 133-157.

Galligan, Ivonne; Tremblay, Manon. 2005. Sharing power. Women, parliament, democracy. Aldershot-Hampshire: Ashgate. 
Guittens, Marianne. 1983. “The elusive paradigm: gender, politics and political behavior: the state of the art". En Ada F. Finifter (ed.), Political Science: the state of the discipline. Washington: APSA, 471-499.

Guzmán, Virginia. 2003. Gobernabilidad democrática y género, una articulación posible. Santiago: Comisión Económica para América Latina (CEPAL).

Guzmán, Claudia; Dougnac, Paulette. 2005. “Perfil estadístico: ¿cómo es la nueva mujer?". El Mercurio, 6 de marzo de 2005, D23.

Hawkesworth, Mary. 2005. “Engendering Political Science: an inmmodest proposal". Politics \& Gender 1 (1): 140-156.

Kelly, Rita Mae; Williams, Linda M.; Fisher, Kimberly. 1994. “Women \& Politics: an assessment of its role within the discipline of Political Science". Women and Politics 14 (4): 3-18.

Kenney, Sally J. 1995. “Women, feminism, gender and law in Political Science: ruminations of a feminist academic". Women and Politics 15 (3): 43-69.

Khtar, P.; P. Fawcett; T. Legrand; D. Marsh; C. Taylor. 2005. "Women in the Political Science profession", PSA News 16, 1 (1), 28.

Lechner, Norbert. 1990. Las condiciones políticas de la Ciencia Política en Chile. Santiago: FLACSO.

Lovenduski, J. 1998. "Gendering research in Political Science". Annual Review of Political Science 1: 333-356.

Matthews, Lanethea; Andersen, Kristi. 2001. “A gender gap in publishing? Women's representation in edited Political Science books?". PS: Political Science E Politics 34 (1): 143-148.

Mackay, Fiona. 2004. "Gender and political representation in the UK". British Journal of Political and International Relations 6: 99-120.

Mazur, Amy G. 2004. "Feminist comparative policy: leading european Political Science into the future". European Political Science, spring, issue 3.2.

Mori. 2006. "Informe de prensa: encuesta MORI. Evaluación de las mujeres en política", Santiago.

Newton, K.; Vallés, Josep M. 1990. “Introduction: Political Science in Western Europe, 1960-1990". European Journal of Political Research 18: 227-240.

Puryear, Jeffrey A. 1994. Thinking politics: intellectuals and democracy in Chile, 1973-1988. Baltimore and London: The Johns Hopkins University Press.

Randall, Vicky. 1991. "Feminism and political analysis". Political Studies 39 (3): 513-532.

Rehren, Alfredo; Fernández, Marco. 2005. “La evolución de la Ciencia Política en Chile: un análisis exploratorio (1980-2000)". Revista de Ciencia Política 25 (1): 40-55.

Ríos, Marcela et al. 2003. ¿Un nuevo silencio feminista? La transformación de un movimiento social en el Chile postdictadura. Santiago, CEM: Editorial Cuarto Propio.

Sarkees, Meredith R.; McGlen, Nancy E. 1992. "Confronting barriers: the status of women in Political Science". Women and Politics 12 (4): 43-85. 
Schkolnik, Mariana. 2004. “¿Por qué es tan increíblemente baja la tasa de participación de las mujeres en Chile?", Documentos En Foco. Santiago: Expansiva.

Uriarte, Edurne. 1997. “Estudios de mujeres y política en España”. En Edurne Uriarte y Arantxa Elizondo (eds.), Mujeres en política. Barcelona: Editorial Ariel, 9-32.

Vianello, M.; G. Moore. 2000. Gendering elites: economics and political leadership in 27 industrialised societies. London: Macmillan Press Ltd.

Waylen, Georgina. 2000. "Gender and democratic politics: a comparative analysis of consolidation in Argentina and Chile". Journal of Latin American Studies 32 (3): 765-793. 